\title{
Identification of driver genes and key pathways of prolactinoma predicts the therapeutic effect of genipin
}

\author{
SHENG ZHONG ${ }^{1-3 *}$, BO WU ${ }^{2,4^{*}}$, XINHUI WANG ${ }^{2}$, DANDAN SUN $^{2}$, DAQUN LIU $^{2}$, SHANSHAN JIANG ${ }^{5}$, \\ JUNLIANG GE $^{2}$, YUAN ZHANG ${ }^{2}$, XINRUI LIU ${ }^{1}$, XIAOLI ZHOU ${ }^{6}$, RIHUA JIN ${ }^{1}$ and YONG CHEN ${ }^{1}$ \\ ${ }^{1}$ Department of Neurosurgery, The First Hospital of Jilin University; ${ }^{2}$ Clinical College, Jilin University, Changchun, \\ Jilin 130012, P.R. China; ${ }^{3}$ Department of Surgery, Harvard Medical School, Harvard University, Boston, \\ MA 02138, USA; ${ }^{4}$ Department of Orthopedics, The First Hospital of Jilin University; ${ }^{5}$ College of Pharmacy, Jilin University; \\ ${ }^{6}$ Department of Cell Biology, Basic Medical College, Jilin University, Changchun, Jilin 130012, P.R. China
}

Received October 12, 2018; Accepted April 11, 2019

DOI: $10.3892 / \mathrm{mmr} .2019 .10505$

\begin{abstract}
The purpose of the present study was to identify the potential targets and markers for diagnosis, therapy and prognosis in patients with prolactinoma at the molecular level and to determine the therapeutic effects of genipin in prolactinoma. The gene expression profiles of GSE2175, GSE26966 and GSE36314 were obtained from the Gene Expression Omnibus database. The differentially expressed genes (DEGs) were identified after comparing between gene expression profiles of the prolactinoma tissues and normal tissues. Then, Gene Ontology (GO), Kyoto Encyclopedia of Genes and Genomes (KEGG) analysis and protein-protein interaction (PPI) network analysis were conducted. In addition, in vitro, scratch assay, colony-forming assay, Cell Counting Kit 8 (CCK8) assay and flow cytometry were performed to verify the functional effects of genipin. An aggregate of 12,695, 3,847 and 5,310 DEGs were identified from GSE2175, GSE26966 and GSE36314, respectively. The results of GO and KEGG
\end{abstract}

Correspondence to: Dr Rihua Jin or Dr Yong Chen, Department of Neurosurgery, The First Hospital of Jilin University, 71 Xinmin Street, Changchun, Jilin 130012, P.R. China

E-mail: jinrihua_jdyy@126.com

E-mail: chenyong12345_jdyy@126.com

*Contributed equally

Abbreviations: CALM1, calmodulin 1; PKG, cGMP-protein kinase G; DEGs, differentially expressed genes; DAVID, Database for Annotation, Visualization and Integrated Discovery; EGR1, early growth response 1; GEO, Gene Expression Omnibus; GO, gene ontology; GSEA, gene set enrichment analysis; GnRH, gonadotropin-releasing hormone; KEGG, Kyoto Encyclopedia of Genes and Genomes; MAPK1, mitogen-activated protein kinase 1; PPI, protein-protein interaction; STRING, Search Tool for Retrieval of Interacting Genes

Key words: bioinformatics, brain science, genipin, prognosis, prolactinoma analysis showed that the DEGs significant and important for prolactinoma were mostly involved with 'spindle pole' and 'oocyte meiosis'. A total of 20 genes were selected as hub genes with high degrees after PPI network analysis, including mitogen-activated protein kinase 1 (MAPK1), MYC, early growth response 1 (EGR1), Bcl2 and calmodulin 1 (CALM1). CCK8 assay, colony-forming assay and scratch assay were performed to verify the anti-prolactinoma effect of genipin. The results of flow cytometry showed that apoptosis was increased by genipin. MAPK1, MYC, EGR1, Bcl2 and CALM1 were screened as main hub genes. Genipin upregulated the expression level of EGR1 and p21 (downstream mediator of EGR1) and EGR1, inhibited the proliferation and migration of prolactinoma cells. Genipin is a promising drug for treatment of patients with prolactinoma.

\section{Introduction}

Prolactinoma is one of the most common central nervous system tumors, which is derived from the hormone secreting epithelial cells in the anterior of the pituitary gland (1). According to the 2016 World Health Organization classification of tumors, prolactinomas are grade I/II (2), which are considered to be benign tumors. In addition, prolactinoma is one of the functional pituitary tumors, which are classified into adrenocorticotropic hormone-secreting pituitary adenoma, growth hormone-secreting pituitary adenoma and prolactinoma (3).

Recently, imaging has become important in the diagnosis of prolactinoma. As the most important tool for imaging diagnosis of prolactinoma, MRI can clearly display the size, shape and position of prolactinoma, as well as its interaction with surrounding structures. Medicinal therapy has become the first choice treatment for prolactinoma, with surgery coming second and other treatments, such as gene therapy, molecular therapeutics, chemotherapeutics, radiotherapy and physiotherapy, used as adjunctive therapy. The dopamine agonists (DAs) are the best medicinal treatment of prolactinoma and can reduce the secretion of prolactin: $70-90 \%$ of patients with microadenomas have normalized prolactin levels after DAs treatment, with menstruation 
resuming, lactation ceasing, fertility restored and shrinkage of the tumor (4). However, its side effects and adverse reactions cannot be ignored: $\leq 4 \%$ of properly regulated patients with prolactinoma can develop acromegaly according to a previous study (5). A recent study noted that cabergoline, one type of DA, increases the risk of valvular heart disease. In addition, there are also some studies indicating that cabergoline is associated with pituitary apoplexy after initiation of cabergoline therapy $(6,7)$. Selective resection of prolactinoma is another common treatment method that results in the symptoms relief after surgery in the majority of patients. Although prolactinomas can be removed by a frontal or a butterfly pathway, it is often difficult to completely excise and postoperative serum prolactin levels are difficult to recover. A previous study reported that $40-80 \%$ surgeries will result in temporary improvement and half of them will relapse (8). Thus, a novel treatment strategy with improved efficacy or fewer side effects is needed.

Recently, research has focused increasingly on the initiation, progression and metastasis of prolactinoma using bioinformatics methods and microarray technology, aiming to reveal the genetic alteration and molecular mechanisms in prolactinoma (9-11). For instance, Zhang et al (12) showed that upregulated solute carrier family 2 , facilitated glucose transporter member 11 and chromogranin B (CHGB) may be important in prolactinoma progression. Faraoni et al (13) also suggested that the transforming growth factor $\beta 1$ system may be involved in prolactinoma progression. However, the number of studies on the explicit molecular mechanisms is small, and there is a limited and shallow understanding of prolactinoma. Therefore, further study of the molecular mechanisms of prolactinoma is urged, particularly the identification of differentially expressed genes (DEGs) and key pathways. In the present study, early growth response 1 (EGR1) was found to have a significant role in the occurrence and progression of prolactinoma by complete bioinformatics analysis, while genipin was reported as an effective targeted drug for EGR1 as it could upregulate the expression level of EGR1 and p21 (downstream mediator of EGR1), and was applied to inhibit gastric cancer in a previous report (14). Therefore, it is hypothesized that genipin may also be able to treat prolactinoma by targeting EGR1.

Bioinformatics is a technology that comprehensively applies biology, computer science and information technology to analyze numerous complex biological data. Bioinformatics combined with microarray has been broadly used to identify genetic alterations during tumorigenesis (15). In the present study, three mRNA microarray datasets were selected to identify DEGs between prolactinoma tissues and normal pituitary tissues. Gene ontology (GO) and Kyoto Encyclopedia of Genes and Genomes (KEGG) analysis were subsequently conducted. Then, a Cell Counting Kit 8 (CCK8) assay, colony-forming assay, scratch assay and flow cytometry were performed to verify the anti-prolactinoma effect of genipin. In brief, the present study aimed to provide data to determine the occurrence and progression mechanism of the prolactinoma and identify precise, promising targets for the treatment of patients with prolactinoma as well as validation of the anti-prolactinoma effect of genipin. The framework of the present study is outlined in Fig. 1.

\section{Materials and methods}

Microarray data. Three human gene expression profiles (GSE2175, GSE26966 and GSE36314) (16-18) were obtained from the Gene Expression Omnibus (GEO; ncbi.nlm.nih. gov/geo/) database in September 2017. GSE2175 contained one normal pituitary sample and one prolactinoma sample. GSE26966 consisted of nine normal pituitary samples and 14 human prolactinoma samples. GSE36314 included three normal pituitary samples and four prolactinoma samples.

Identification of DEGs. The analysis of three files was performed in September 2017, using the GeneSpring GX software (version 11.5; Agilent Technologies, Inc.), which provides powerful, accessible tools for intuitive data analysis and visualization, and this software has been extensively applied in bioinformatics analysis (19). Through the analysis of the three sequences, the DEGs between prolactinoma samples and normal samples were identified. Hierarchical clustering analysis and principal component analysis were applied to determine the probe quality control in GeneSpring. Probes with intensity values below the 20th percentile were filtered out using the 'filter probesets by expression' option. Classical t-test was used to identify DEGs, with a two-fold cutoff and $\mathrm{P}<0.01$ applied to determine statistical significance, the samples size was increased to reduce false positives and false negatives. Then, a Venn plot was created using DEGs of the three datasets (bioinformatics.psb.ugent.be/webtools/Venn; version 2.1).

GO and pathway enrichment analysis of DEGs. The Database for Annotation, Visualization and Integrated Discovery (DAVID, version 6.8) is an online program that provides a comprehensive set of functional annotation tools for researchers to understand biological meaning behind plenty of genes (20). GO analysis is a method that analyzes gene function, cell component and biological process. KEGG is a base for gene function analysis and genomic information link. The two were conducted online based on DAVID, and the annotation tool applied. Gene Set Enrichment Analysis (GSEA; broadinstitute.org/gsea/index.jsp) was performed (also in September, 2017) to determine which sets of genes exhibited statistical significance.

Protein-protein interaction (PPI) network construction and module selection. A PPI network of DEGs was constructed through the Search Tool for the Retrieval of Interacting Genes (STRING) database. In the present study, Molecular Complex Detection was performed based on Cytoscape (version 3.4.0) software to reveal modules of the PPI network (21). GO and KEGG pathway enrichment analysis were performed for genes in the modules. The Molecular Complex Detection (MCODE) application was applied to screen modules of PPI network in Cytoscape with degree cutoff $=2$, node score cutoff $=0.2$.

Cell lines. A rat pituitary tumor cell line (GH3) was obtained from the American Type Culture Collection and mouse pituitary tumor cell line (GT1-1) from BeNa Culture Collection. The cell lines were cultured in DMEM (HyClone; GE Healthcare Life Sciences) supplemented with 10\% FBS (Gibco; Thermo 


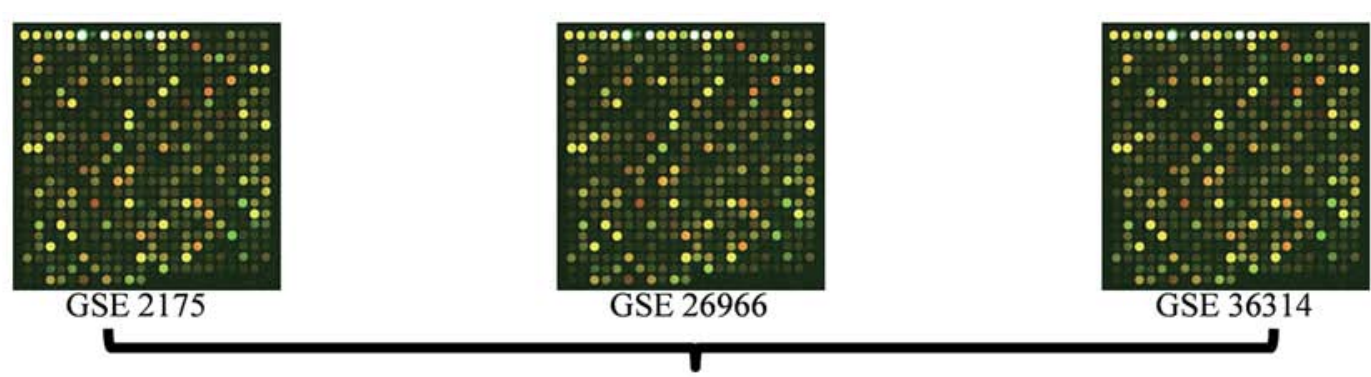

Identification of DEGs


PPI analysis
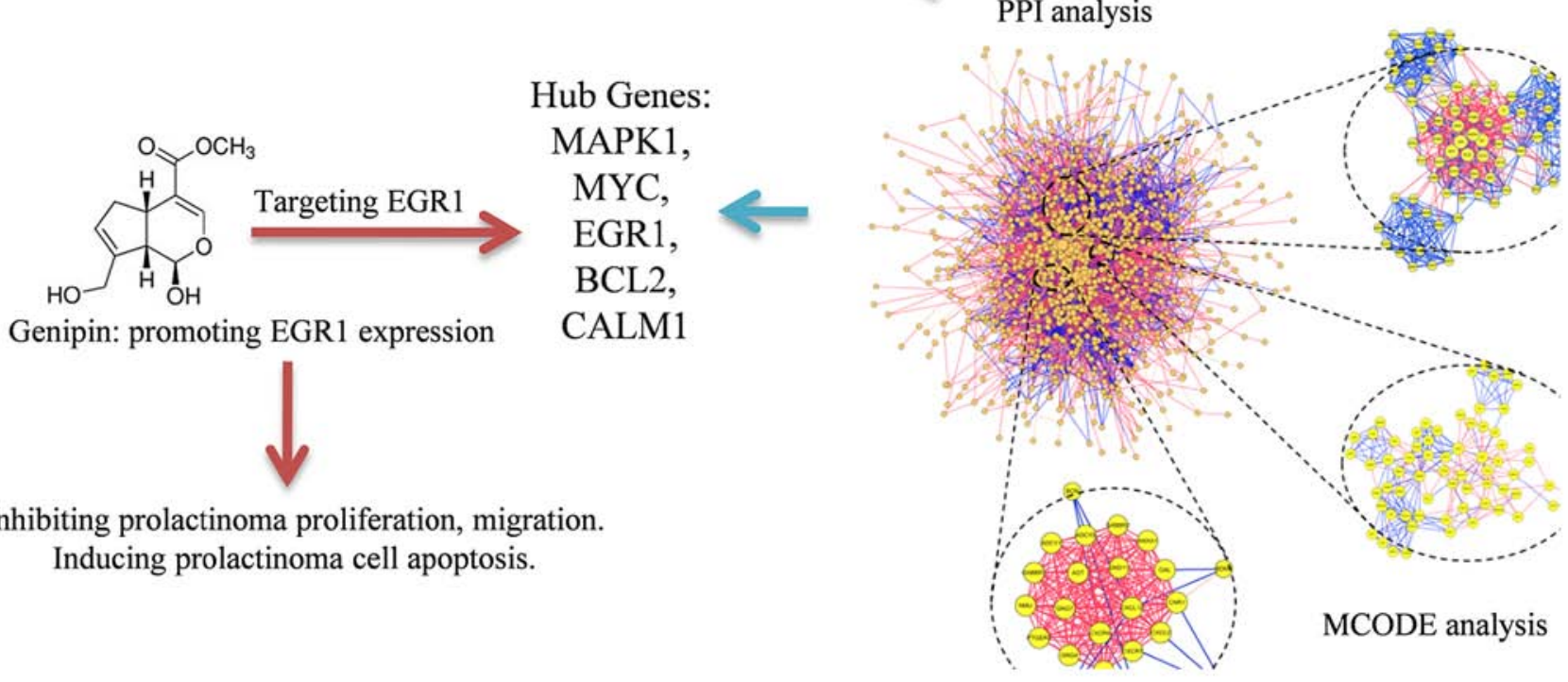

Figure 1. Study framework. The top three images were selected to represent information of tissue datasets from the Gene Expression Omnibus database. DEG, differentially expressed gene; GSEA, gene set enrichment analysis; GO, gene ontology; KEGG, Kyoto Encyclopedia of Genes and Genomes; EGR, early growth response 1; MAPK, mitogen-activated protein kinase; CALM, calmodulin 1; PPI, protein-protein interaction.

Fisher Scientific, Inc.). An atmosphere of $5 \% \mathrm{CO}_{2}$ and $95 \%$ air at $37^{\circ} \mathrm{C}$ was maintained for the cultivation of the cell lines. Genipin was purchased from Apexbio, Inc.

CCK8 assay. A total of 2,000 cells were seeded in 96-well plates and cultured in DMEM including 10\% FBS for 6 days. Then, $10 \mu \mathrm{l}$ CCK- 8 was added into each well and the plate was incubated at $37^{\circ} \mathrm{C}$ for $2 \mathrm{~h}$. The viability of cells was determined at $24 \mathrm{~h}$. The cell viability was determined by measuring absorbance at $450 \mathrm{~nm}$ on an ELX800 UV universal microplate reader (BioTek Instruments Inc.). The experiments were repeated three times.

Colony-forming assay. The prolactinoma cells were cultured in Petri dishes with the density of 50 cells $/ \mathrm{cm}^{2}$. They were cultivated for $24 \mathrm{~h}$ in vitro and then treated with different doses of genipin $(0,0.5$ and $2.0 \mu \mathrm{mol} / \mathrm{l})$. Colonies were counted and described according to Franken et al after 10 days in vitro growth (22). Then, colonies were rinsed with PBS, fixed in $4 \%$ paraformaldehyde at $4^{\circ} \mathrm{C}$ for $30 \mathrm{~min}$, stained with $5 \%$ crystal violet for $10 \mathrm{~min}$ at room temperature and rinsed twice with water. This experiment was repeated three times.

In vitro scratch assay. The GT1-1 cells were cultured to confluence on 24-well Permanox plates. A $10 \mu$ pipette tip across each well was used to create a consistent cell-free area which the loose cells were washed out gently using DMEM. Afterwards, the cells were exposed to different doses of genipin $(0,0.5$ and $2.0 \mu \mathrm{mol} / \mathrm{l})$. After the scratch and at 12 , $24 \mathrm{~h}$, images of the scraped area were captured using phase 
contrast microscopy. The remaining wounded area and the scratch width at six different points per image were measured. This experiment was repeated three times.

Flow cytometry. Prolactinoma cells in the log growth phase were seeded into 6 -well plates with a density of $2 \times 10^{5}$ cells/well and the cells were treated with different doses of genipin $(0,0.5$ and $2.0 \mu \mathrm{mol} / \mathrm{l})$. Following culture for $48 \mathrm{~h}$, the cells were harvested using accutase detachment solution (Sigma-Aldrich; Merck KGaA) and Annexin-V-FITC/propidium labeling was conducted using Annexin-V-FITC/PI apoptosis detection kit (KGI Biotechnology. Ltd.) according to the manufacturer's protocol. The flow cytometer was used to detect the stained cells and the results analyzed with the FACSDiva version 6.2 (BD Biosciences). This experiment was repeated three times.

Statistical analysis. All statistical data were entered into SPSS version 18.0 (SPSS Inc.) for analysis. One-way analysis of variance was performed to analyze quantitative data and Tamhane's $\mathrm{T} 2$ was used to conduct post hoc testing. Data are presented as mean \pm standard deviation. $\mathrm{P}<0.05$ was considered to indicate a statistically significant difference.

\section{Results}

Identification of DEGs. An aggregate of 12,695 DEGs were identified from GSE2175, of which 8,314 were upregulated and 4,381 were downregulated. A total of 3,847 DEGs were identified in GSE26966, among which 1,766 were upregulated and 2,081 were downregulated. A total of 5,310 DEGs were picked up from GSE36314, which 2,246 genes were upregulated and 3,064 genes were downregulated. Venn plot demonstrated that there were 1,201 common DEGs among the three datasets (Fig. 2A and Table I). Among them, 570 mutual upregulated genes and 631 mutual downregulated genes were identified. Heat maps of the DEGs in GSE26966 and GSE36314 that perform the most important role are presented in Fig. 2B and C.

Functional and pathway enrichment analysis. To further investigate the function of identified DEGs, the mutual downregulated and upregulated DEGs were entered into DAVID for GO and KEGG pathways analysis. The GO analysis and KEGG analysis of DEGs are presented in Table II. For biological process, upregulated DEGs were significantly enriched in 'positive regulation cyclic nucleotide metabolic process', 'response to hormone' and 'positive regulation of cyclic-nucleotide phosphodiesterase activity' terms, while downregulated DEGs were enriched in 'peptidyl-tyrosine phosphorylation', 'signal transduction' and 'positive regulation of cell cycle'. For cell component, the upregulated genes were mainly enriched in the 'associated cytosol', 'nucleoplasm' and 'spindle pole', and the downregulated DEGs enriched in 'cytoplasm', 'receptor complex' and 'integral component of plasma membrane' terms. For molecular function, the upregulated DEGs enriched in 'protein binding', 'N-terminal myristoylation domain binding' and 'phospholipase binding' terms; the downregulated DEGs tended to enrich in 'protein binding', 'protein tyrosine kinase activity' and 'transmembrane receptor protein tyrosine kinase activity' terms. Table II also presents the most significant enriched pathways of the mutual upregulated DEGs and downregulated DEGs according to KEGG analysis. The upregulated DEGs were enriched in the terms 'glioma', 'oocyte meiosis' and 'cGMP-PKG signaling pathway', while the downregulated DEGs were mostly involved in pathways including 'cancer', 'focal adhesion' and 'PI3K-Akt signaling pathway' (Fig. 2D and E). In addition, the results of GSEA analysis indicated that the expression profiles of prolactinomas were mainly enriched in 'calcium signaling pathway' and 'Parkinson's disease' (Fig. 2F and G).

Module screening from the PPI network. The PPI network of DEGs consisted of 1,142 nodes and 7,278 edges. Degrees $>72$ were set as the cut-off criterion. A total of 20 genes were selected as hub genes: MAPK1, JUN, MYC, FOS, EGR1, Bcl2, IL6, CALM1, IL8, STAT3, ESR1, RPS27A, ACACB, CD44, INSR, ERBB2, PPP2CA, CDK2, PTPRC and SMAD3 (Table III). Among those genes, the node degree of Mitogen-activated protein kinase 1 (MAPK1) was the highest, at 183 . The top three significant modules were selected (Fig. 3). Functional annotation of the modules genes is shown in Table IV. The genes of module 1 were associated with cellular structure, including 'endoplasmic reticulum lumen', 'extracellular matrix organization' and 'collagen trimer'. Genes of module 2 were associated with 'cancer', 'GABAergic synapse' and 'morphine addiction'. Genes of module 3 associated with 'type I interferon signaling pathway', 'nucleoplasm' and 'PI3K-Akt signaling pathway'.

Genipin reduces proliferation of prolactinoma cells. CCK8 assay was performed to evaluate the effects of genipin on cell survival. The results revealed that the viability of cells in cell lines GH3 and GT1-1 were significantly decreased by genipin, as in Fig. 4A. For further confirmation, a colony-forming assay was conducted to define the effects of genipin in prolactinoma cells. As demonstrated in Fig. 4B and C, there were fewer and smaller clonogenicities in the genipin group compared with the control group, and the percentage of clone formation in the control was significantly higher than the drug groups ( 0.5 and $2 \mu \mathrm{mol} / 1 ; \mathrm{P}<0.05$ ), and the percentage of clone formation was found to be significantly different between the two different drug dose groups.

Genipin induces migration of prolactinoma cells. A scratch assay was performed to verify the effect of genipin on invasion and migration of prolactinoma cells, with the widths of scratched areas recorded 0,12 and $24 \mathrm{~h}$ following the scratch. As demonstrated in Fig. 4D and E, the widths of scratched were clearly smaller after $24 \mathrm{~h}$ in the control group, but only slightly decreased in genipin group, while after $24 \mathrm{~h}$, it was found that the wounds in control group were also clearly smaller compared with the genipin group. With time, the wound widths decreased in the control and the genipin groups, and the control group showed a significant decrease compared with the genipin group.

Genipin induces apoptosis of prolactinoma cells. Flow cytometry was performed to clarify the mechanism of genipin inhibition in prolactinoma cells and the prolactinoma cells were measured following culture with different doses of genipin for 48 h. Fig. 5A and B demonstrate that the percentage of GH3 
Table I. Venn plot analysis results of DEGs among the three datasets.

\begin{tabular}{lcl}
\hline Name & Total DEGs & \multicolumn{1}{c}{ Elements } \\
\hline $\begin{array}{l}\text { GSE2175, GSE26966 } \\
\text { and GSE36314 }\end{array}$ & 1,201 & CREB3L1, SMARCD3, RALYL, BBOX1, CXCR4 ... \\
GSE2175 and GSE26966 & 1,378 & MSRB1, ELMO2, PKNOX2, CLK4, IRAK1 ... \\
GSE2175 and GSE36314 & 3,653 & MMP2, SAMD4A, GPR98, KCNG1, DECR1 ... \\
GSE26966 and GSE36314 & 118 & TMSB10, KCNA6, NEFH, ID1, CGA... \\
GSE2175 & 6,463 & AACS, RPS11, PNMA1, HAUS2, A4GNT ... \\
GSE26966 & 1,150 & CLMP, CD99P1, LOC100287896, TMC4, LINC01279... \\
GSE36314 & 338 & RPS18, ZG16, SERPINF1, NUDT1, LOC101930303 /// TRIM16 /// TRIM16L
\end{tabular}

DEG, differentially expressed gene. /// genes sharing one genetic locus.

A

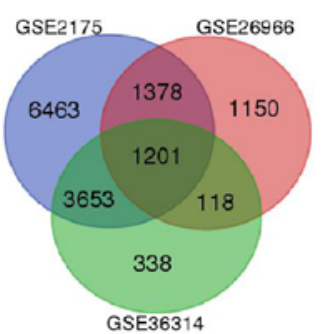

GSE36314
B



TYPE

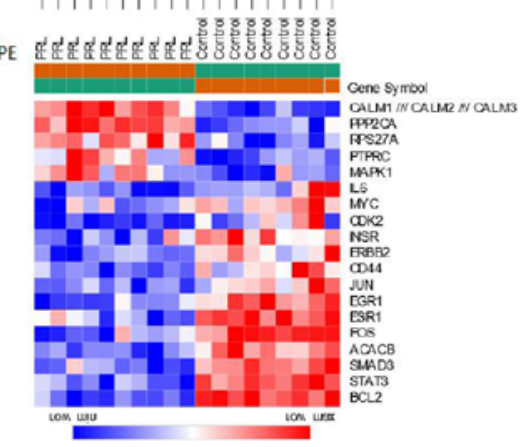

$\mathrm{C}$

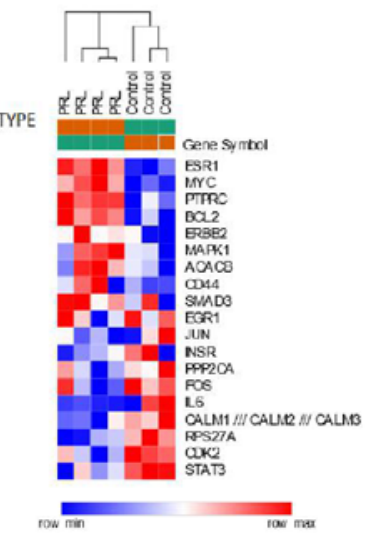

$\mathrm{D}$

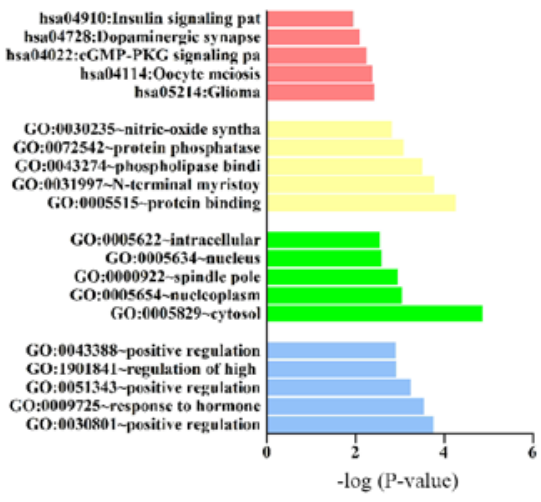

F

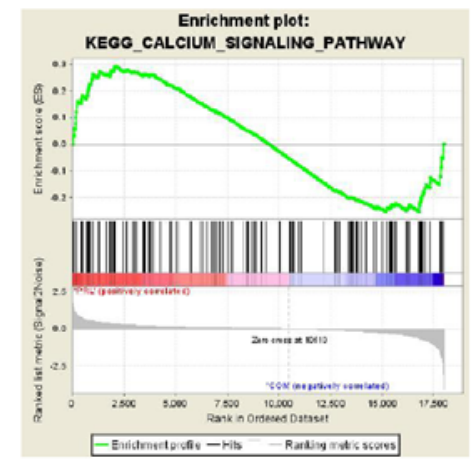

E

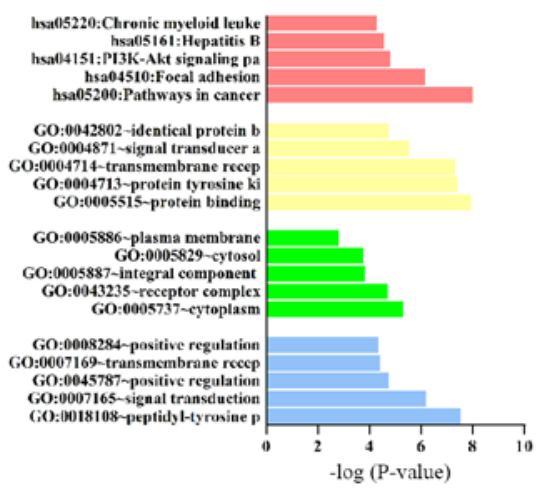

G



Figure 2. (A) Venn plot of DEGs among the three datasets. (B) DEGs expression heat map of GSE26966. (C) DEGs expression heat map of GSE36314; red represents upregulated genes and blue downregulated genes). (D) Functional and pathway enrichment analysis of upregulated genes among the three datasets. (E) Functional (GO) and pathway (KEGG) enrichment analysis of downregulated genes among the three datasets. The results of GO include biological processes, cell component, and molecular function, marked with different colors, as well as results of KEGG. Gene set enrichment analysis results among the three datasets for $(\mathrm{F})$ 'calcium signaling pathway' and $(\mathrm{G})$ 'Parkinson's disease'. The raw data were downloaded from Gene Expression Omnibus. DEG, differentially expressed gene; PRL, prolactinoma; GO, gene ontology; KEGG, Kyoto Encyclopedia of Genes and Genomes. 
Table II. Functional and pathway enrichment analysis of upregulated and downregulated genes among three datasets (the primitive data downloaded from Gene Expression Omnibus database).

A, Upregulated

\begin{tabular}{|c|c|c|c|c|}
\hline Category & Term & Count & $\%$ & P-value \\
\hline GOTERM_BP_DIRECT & $\begin{array}{l}\text { GO:0030801 positive regulation of cyclic nucleotide } \\
\text { metabolic process }\end{array}$ & 3 & 0.012881618 & $1.72 \times 10^{-4}$ \\
\hline GOTERM_BP_DIRECT & GO:0009725 response to hormone & 5 & 0.021469363 & $2.81 \times 10^{-4}$ \\
\hline GOTERM_BP_DIRECT & $\begin{array}{l}\text { GO: } 0051343 \text { positive regulation of cyclic-nucleotide } \\
\text { phosphodiesterase activity }\end{array}$ & 3 & 0.012881618 & $5.68 \times 10^{-4}$ \\
\hline GOTERM_BP_DIRECT & $\begin{array}{l}\text { GO: } 1901841 \text { regulation of high voltage-gated calcium } \\
\text { channel activity }\end{array}$ & 3 & 0.012881618 & $1.18 \times 10^{-3}$ \\
\hline GOTERM_BP_DIRECT & GO:0043388 positive regulation of DNA binding & 4 & 0.017175491 & $1.23 \times 10^{-3}$ \\
\hline GOTERM_CC_DIRECT & GO:0005829 cytosol & 45 & 0.193224269 & $1.38 \times 10^{-5}$ \\
\hline GOTERM_CC_DIRECT & GO:0005654 nucleoplasm & 35 & 0.150285543 & $8.99 \times 10^{-4}$ \\
\hline GOTERM_CC_DIRECT & GO:0000922 spindle pole & 6 & 0.025763236 & $1.10 \times 10^{-3}$ \\
\hline GOTERM_CC_DIRECT & GO:0005634 nucleus & 55 & 0.236162995 & $2.63 \times 10^{-3}$ \\
\hline GOTERM_CC_DIRECT & GO:0005622 intracellular & 20 & 0.085877453 & $2.86 \times 10^{-3}$ \\
\hline GOTERM_MF_DIRECT & GO:0005515 protein binding & 89 & 0.382154665 & $5.46 \times 10^{-5}$ \\
\hline GOTERM_MF_DIRECT & GO:0031997 N-terminal myristoylation domain binding & 3 & 0.012881618 & $1.68 \times 10^{-4}$ \\
\hline GOTERM_MF_DIRECT & GO:0043274 phospholipase binding & 4 & 0.017175491 & $3.12 \times 10^{-4}$ \\
\hline GOTERM_MF_DIRECT & GO:0072542 protein phosphatase activator activity & 3 & 0.012881618 & $8.26 \times 10^{-4}$ \\
\hline GOTERM_MF_DIRECT & GO:0030235 nitric-oxide synthase regulator activity & 3 & 0.012881618 & $1.53 \times 10^{-3}$ \\
\hline KEGG_PATHWAY & hsa05214: Glioma & 5 & 0.021469363 & $3.70 \times 10^{-3}$ \\
\hline KEGG_PATHWAY & hsa04114: Oocyte meiosis & 6 & 0.025763236 & $4.22 \times 10^{-3}$ \\
\hline KEGG_PATHWAY & hsa04022: cGMP-PKG signaling pathway & 7 & 0.030057109 & $5.62 \times 10^{-3}$ \\
\hline KEGG_PATHWAY & hsa04728: Dopaminergic synapse & 6 & 0.025763236 & $8.28 \times 10^{-3}$ \\
\hline KEGG_PATHWAY & hsa04910: Insulin signaling pathway & 6 & 0.025763236 & $1.23 \times 10^{-2}$ \\
\hline
\end{tabular}

B, Downregulated

\begin{tabular}{|c|c|c|c|c|}
\hline GOTERM_BP_DIRECT & GO:0018108 peptidyl-tyrosine phosphorylation & 13 & 0.050286245 & $2.95 \times 10^{-8}$ \\
\hline GOTERM_BP_DIRECT & GO:0007165 signal transduction & 31 & 0.119913353 & $6.22 \times 10^{-7}$ \\
\hline GOTERM_BP_DIRECT & GO:0045787 positive regulation of cell cycle & 6 & 0.023209036 & $1.74 \times 10^{-5}$ \\
\hline GOTERM_BP_DIRECT & $\begin{array}{l}\text { GO:0007169 transmembrane receptor protein tyrosine } \\
\text { kinase signaling pathway }\end{array}$ & 8 & 0.030945381 & $3.96 \times 10^{-5}$ \\
\hline GOTERM_BP_DIRECT & GO:0008284 positive regulation of cell proliferation & 16 & 0.061890763 & $4.64 \times 10^{-5}$ \\
\hline GOTERM_CC_DIRECT & GO:0005737 cytoplasm & 75 & 0.290112951 & $4.95 \times 10^{-6}$ \\
\hline GOTERM_CC_DIRECT & GO:0043235 receptor complex & 9 & 0.034813554 & $1.96 \times 10^{-5}$ \\
\hline GOTERM_CC_DIRECT & GO:0005887 integral component of plasma membrane & 28 & 0.108308835 & $1.56 \times 10^{-4}$ \\
\hline GOTERM_CC_DIRECT & GO:0005829 cytosol & 50 & 0.193408634 & $1.74 \times 10^{-4}$ \\
\hline GOTERM_CC_DIRECT & GO:0005886 plasma membrane & 55 & 0.212749497 & $1.53 \times 10^{-3}$ \\
\hline GOTERM_MF_DIRECT & GO:0005515 protein binding & 116 & 0.44870803 & $1.13 \times 10^{-8}$ \\
\hline GOTERM_MF_DIRECT & GO:0004713 protein tyrosine kinase activity & 12 & 0.046418072 & $3.50 \times 10^{-8}$ \\
\hline GOTERM_MF_DIRECT & $\begin{array}{l}\text { GO:0004714 transmembrane receptor protein tyrosine } \\
\text { kinase activity }\end{array}$ & 8 & 0.030945381 & $4.77 \times 10^{-8}$ \\
\hline GOTERM_MF_DIRECT & GO:0004871 signal transducer activity & 12 & 0.046418072 & $2.91 \times 10^{-6}$ \\
\hline GOTERM_MF_DIRECT & GO:0042802 identical protein binding & 21 & 0.081231626 & $1.76 \times 10^{-5}$ \\
\hline KEGG_PATHWAY & hsa05200: Pathways in cancer & 23 & 0.088967972 & $9.94 \times 10^{-9}$ \\
\hline KEGG_PATHWAY & hsa04510: Focal adhesion & 15 & 0.05802259 & $6.84 \times 10^{-7}$ \\
\hline KEGG_PATHWAY & hsa04151: PI3K-Akt signaling pathway & 17 & 0.065758935 & $1.57 \times 10^{-5}$ \\
\hline KEGG_PATHWAY & hsa05161: Hepatitis B & 11 & 0.042549899 & $2.67 \times 10^{-5}$ \\
\hline KEGG_PATHWAY & hsa05220: Chronic myeloid leukemia & 8 & 0.030945381 & $5.28 \times 10^{-5}$ \\
\hline
\end{tabular}

GO, gene ontology; BP, biological process; CC, cellular component; MF, molecular function; KEGG, Kyoto Encyclopedia of Genes and Genomes. 
Table III. Hub genes among the three datasets.

\begin{tabular}{lcr}
\hline Gene symbol & Degree & Betweenness \\
\hline MAPK1 & 183 & 0.08796778 \\
JUN & 163 & 0.05703413 \\
MYC & 147 & 0.06479138 \\
FOS & 123 & 0.02616212 \\
EGR1 & 120 & 0.0140681 \\
Bc12 & 119 & 0.0274578 \\
IL6 & 117 & 0.04994624 \\
CALM1 & 116 & 0.06850938 \\
IL8 & 110 & 0.02885198 \\
STAT3 & 108 & 0.02679268 \\
ESR1 & 103 & 0.03289612 \\
RPS27A & 94 & 0.04228441 \\
ACACB & 89 & 0.04358763 \\
CD44 & 88 & 0.02006192 \\
INSR & 84 & 0.01708239 \\
ERBB2 & 81 & 0.01628601 \\
PPP2CA & 77 & 0.02323539 \\
CDK2 & 73 & 0.02347513 \\
PTPRC & 73 & 0.01304584 \\
SMAD3 & 72 & 0.02005951 \\
\hline
\end{tabular}

cells that were defined as non-apoptotic, necrotic, late apoptosis or early apoptosis were $96.0,0.26,0.39$ and $3.31 \%$, respectively, in the control group, 21.8, 1.39, 69.6 and $7.25 \%$ in the low dose group, and 1.72, 2.02, 88.4 and $7.81 \%$ in the high dose group; while the percentage of non-apoptotic, necrotic, late apoptosis or early apoptosis GT1-1 cells were 62.9, 9.92, 15.0 and $12.2 \%$ in the control group, $0.94,0.23,49.7$ and $49.1 \%$ in the low dose group, and $0.69,0.02,42.1$ and $57.2 \%$ in the high dose group, respectively. Fig. 5C and D demonstrates that normal (non-apoptotic) cells were dominant in the control group; in the low and dose groups, the percentage of late and early apoptosis cells was increased.

\section{Discussion}

Prolactinoma is the most common pituitary tumor with a secretory function. Prolactinoma monoclonal adenomas account for $\sim 15 \%$ of primary intracranial neoplasms. Prolactinoma is usually benign, but secretes excess hormones, which may lead to distinct endocrine syndromes, and tumor growth or compressive symptoms (23). Despite the common application treatment with DAs and selective resection of prolactinoma, their side effects and adverse reactions are difficult to ignore. Recently, studies focusing on prolactinoma have been performed $(12,13)$, but the mechanism of prolactinoma occurrence remains poorly understood and further study is required. Therefore, promising therapy targets for prolactinoma and data to illustrate the progression mechanism of prolactinoma are provided in the present study. In addition, genipin is reported as a drug promoting EGR1 expression (14), which was identified as hub gene in the present study. CCK8 assay, colony-forming assay, scratch assay and flow cytometry were performed to verify the anti-prolactinoma effects of genipin.

The present study extracted data from GSE2175, GSE26966 and GSE36314 datasets, and identified 12,695, 3,847 and 5,310 DEGs, respectively. A total of 1,201 mutual DEGs were screened among the three datasets, with 570 mutual upregulated DEGs and 631 mutual downregulated DEGs. These DEGs may have an important role during the pathogenesis of prolactinoma and could be applied as diagnostic or prognosis markers and treatment targets in the future.

GO and KEGG pathway enrichment analysis were performed in order to gain further insight into the molecular mechanism of the prolactinoma occurrence. The upregulated DEGs were mostly associated with 'spindle pole', 'oocyte meiosis' and 'nucleus', which are associated with development of tumors. The downregulated genes were mainly associated with 'signal transduction', 'cytoplasm' and 'receptor complex', which meant the decline of normal cell functions. In addition, it was noted that 'peptidyl-tyrosine phosphorylation gene' decreased, which may be connected with the activation of proto-oncogenes or the inactivation of the tumor suppressor genes. According to the results of KEGG analysis, the upregulated DEGs were mainly enriched in 'cGMP-PKG signaling pathway', 'glioma' and 'oocyte meiosis', while the downregulated DEGs were associated with 'focal adhesion' and 'PI3K-Akt signaling pathway'. A number of studies have demonstrated that disruption of the PKG signaling pathway leads to various pathological changes in the heart, including vascular and ventricular dysfunction, fibrosis and hypertrophy $(24,25)$. The cGMP-PKG signaling pathway has also been reported as having a close association with colon cancer and has been considered as a therapeutic strategy for colon cancer (26). It has been hypothesized that blocking the PKG signaling pathway might be an effective solution for patients with prolactinoma (27). Previous studies have shown that the PI3K-Akt signaling pathway performs a crucial role in the growth, proliferation, metabolism, survival and angiogenesis of cancer cells $(28,29)$. For instance, Wu et al (30) identified that the PI3K-Akt signaling pathway effectively ameliorated gastric tumor development by delaying growth, inducing apoptosis, and inhibiting metastasis and angiogenesis. The present study hypothesized that reduced function of PI3K-Akt signaling pathway may be associated with prolactinoma metastasis and invasion. The GSEA analysis suggested that prolactinoma has a close association with Parkinson's disease and the calcium signaling pathway, which supports the results of GO and KEGG analysis. This indicates that there may be a link between the progression mechanism of prolactinoma and Parkinson's disease, but this requires further investigation.

In order to obtain the hub genes among the identified DEGs, the 1,021 DEGs were analyzed using PPI network according to the STRING database. A total of 24 genes were selected with degrees >72; EGR1, MAPK1, MYC, Bcl2 and calmodulin 1 (CALM1). EGR1, a primary response gene that encodes a zinc finger DNA-binding protein, is reported to be critical in cancer metastasis and tumor invasion (31). This gene was found to be regulated by gonadotropin-releasing hormone $(\mathrm{GnRH})$ to regulate the expression of luteinizing hormone $\beta$ polypeptide, one of the GnRH-responsive genes, and was revealed to be associated with prolactinoma in a previous study (31). Previous 


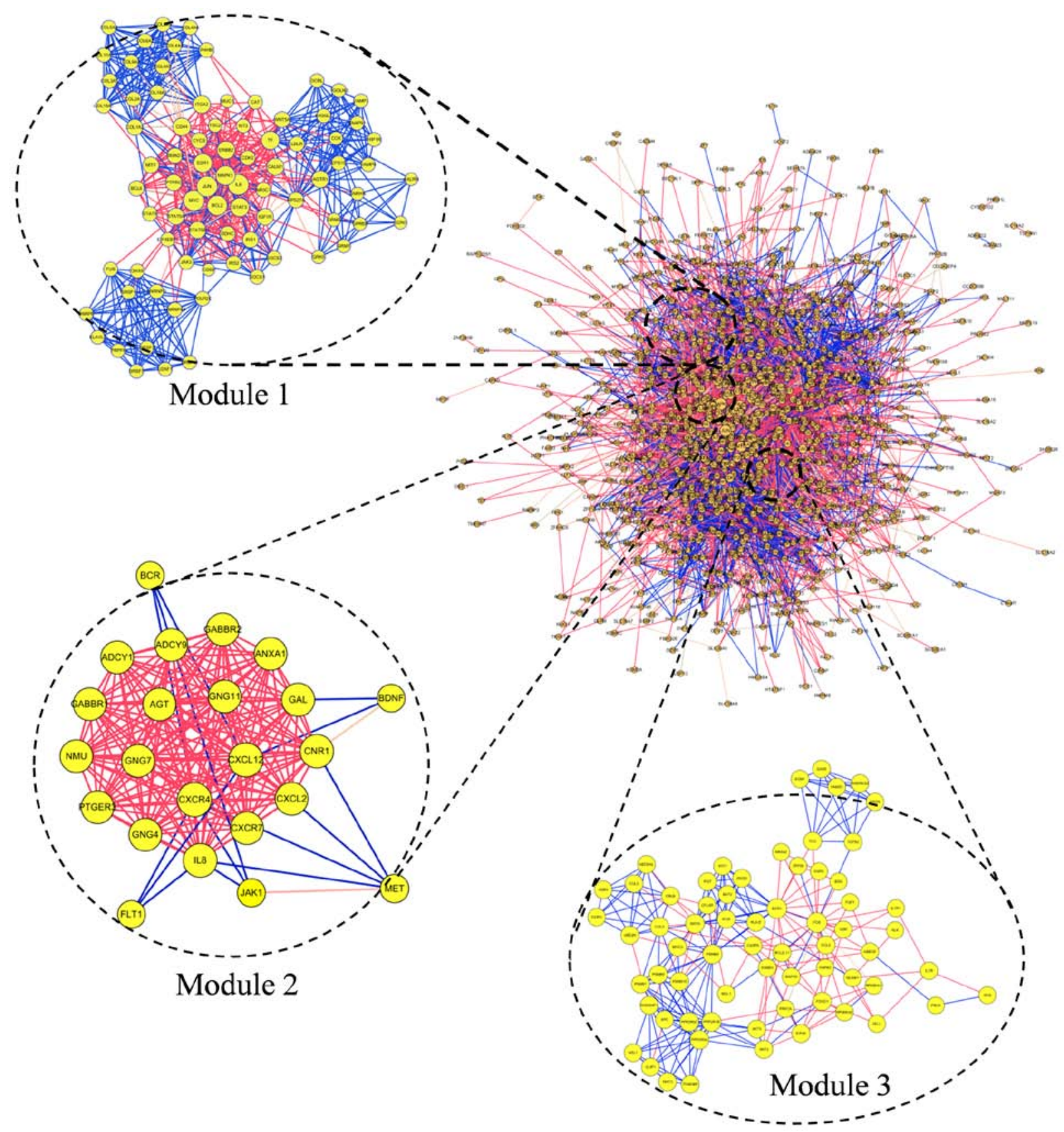

Figure 3. Top three modules from the protein-protein interaction network.

studies have suggested that genipin inhibits the proliferation of various cancer cells by promoting EGR1 function, such as gastric, colon and breast cancer $(32,33)$. Ko et al (14) demonstrated that genipin can induce apoptosis in the AGS human gastric cancer cell via a p53-independent EGR1/p21 signaling pathway. Thus, it was hypothesized that genipin might have the same effect on prolactinoma. MAPK1 interacts with a series of important signaling components and phosphorylation events, which are vital in the process of neoplasia. Extracellular signals are transmitted by MAPK1, which adjusts cell growth, differentiation, proliferation, apoptosis and migration (34). For instance, microRNA-585 can suppress tumor proliferation and migration in gastric cancer by directly targeting MAPK1 (35). In addition, a previous study demonstrated that fulvestrant was effective in suppressing prolactinoma cells by inhibiting the estrogen receptor and MAPK pathway (36). This implies that MAPK1 is a potential treatment target for prolactinoma and the role of MAPK1 in prolactinoma requires further study. MYC, a transcription factor that is overexpressed in cancers, is a proto-oncogen, which is extensively expressed in Burkitt lymphoma (37). MYC was one of hub genes identified in the present study and exhibited abnormal expression compared with normal tissue and prolactinoma tissue; it served an important role in the progression of prolactinoma. Kim et al (38) demonstrated that MYC is involved in preventing immune cells from attacking non-small cell lung cancer cells. A previous study noted that translocations of MYC contributed to lymphoma, especially involving $\mathrm{Bcl} 2$, and indicated a poor prognosis (39). High serum LDH levels are often associated with malignant tumor, which may be related 
Table IV. Functional and pathway enrichment analysis of the modules genes.

A, Module 1

\begin{tabular}{lccll}
\hline Term & Count & P-value & $\begin{array}{c}\text { False } \\
\text { discovery rate }\end{array}$ & \multicolumn{1}{c}{ Genes } \\
\hline $\begin{array}{l}\text { GO:0005788 endoplasmic reticulum } \\
\text { lumen (CC) }\end{array}$ & 8 & $4.18 \times 10^{-7}$ & $4.93 \times 10^{-4}$ & $\begin{array}{l}\text { P4HB, COL9A2, COL2A1, COL16A1, } \\
\text { GO:0030198 extracellular matrix }\end{array}$ \\
$\begin{array}{l}\text { organization (BP) } \\
\text { GO:0005581 collagen trimer (CC) }\end{array}$ & 8 & $8.27 \times 10^{-7}$ & $1.23 \times 10^{-3}$ & COL9A2, CD44, COL2A1, COL16A1, \\
& 6 & $3.06 \times 10^{-6}$ & $3.61 \times 10^{-3}$ & COL11A1, COL5A2, COL4A6, COL4A5 \\
& & & & COL4A6, COL4A5 \\
\hline
\end{tabular}

B, Module 2

\begin{tabular}{|c|c|c|c|c|}
\hline hsa05200: Pathways in cancer (KEGG) & 11 & $4.30 \times 10^{-9}$ & $4.68 \times 10^{-6}$ & $\begin{array}{l}\text { ADCY1, PTGER3, BCR, ADCY9, CXCR4, } \\
\text { MET, JAK1, GNG11, GNG4, CXCL12, GNG7 }\end{array}$ \\
\hline hsa04727: GABAergic synapse (KEGG) & 7 & $3.22 \times 10^{-8}$ & $3.50 \times 10^{-5}$ & $\begin{array}{l}\text { ADCY1, ADCY9, GABBR1, GNG11, } \\
\text { GABBR2, GNG4, GNG7 }\end{array}$ \\
\hline hsa05032: Morphine addiction (KEGG) & 7 & $4.87 \times 10^{-8}$ & $5.29 \times 10^{-5}$ & $\begin{array}{l}\text { ADCY1, ADCY9, GABBR1, GNG11, } \\
\text { GABBR2, GNG4, GNG7 }\end{array}$ \\
\hline
\end{tabular}

C, Module 3

\begin{tabular}{|c|c|c|c|c|}
\hline $\begin{array}{l}\text { GO:0060337 type I interferon signaling } \\
\text { pathway (BP) }\end{array}$ & 7 & $4.29 \times 10^{-9}$ & $5.96 \times 10^{-6}$ & $\begin{array}{l}\text { EGR1, IFI27, IFIT1, ISG15, BST2, PSMB8, } \\
\text { ISG20 }\end{array}$ \\
\hline GO:0005654 nucleoplasm (CC) & 18 & $5.93 \times 10^{-6}$ & $6.46 \times 10^{-3}$ & $\begin{array}{l}\text { PRKCA, ITGB3BP, IER2, EGR1, PPP2R5C, } \\
\text { FOXO1, SMC3, PSMB8, } \\
\text { PSMB9, ISG20, CUL2, RPS6KA1, ISG15, } \\
\text { CASP8, MAPK9, FGF1, AKT3, AKT2 }\end{array}$ \\
\hline $\begin{array}{l}\text { hsa04151: PI3K-Akt signaling } \\
\text { pathway (KEGG) }\end{array}$ & 9 & $9.02 \times 10^{-6}$ & $1.05 \times 10^{-2}$ & $\begin{array}{l}\text { GH2, PRKCA, PRLR, PPP2R5A, PPP2R5C, } \\
\text { FGF1, IL7R, AKT3, AKT2 }\end{array}$ \\
\hline
\end{tabular}

GO, gene ontology; CC, cellular component; BP, biological process; KEGG, Kyoto Encyclopedia of Genes and Genomes.

to prolactinoma and play an important role in the diagnosis of prolactinoma (40). Bcl2, the most important inhibitor of apoptosis in the $\mathrm{Bcl}$ family, is associated with the development and progression of the majority of cancers. It inhibits cell apoptosis and resists other forms of cell death leading to an increase in the number of cells, which has a positive effect on the growth of tumors (41). A previous study noted that the expression level of $\mathrm{Bcl} 2$ could be changed apparently by fulvestrant during medical treatment (42). Geng et al (43) also demonstrated that bromocriptine can induce autophagy-dependent cell death in pituitary adenomas by decreasing $\mathrm{Bcl} 2$. Thus, it was hypothesized that $\mathrm{Bcl} 2$ may be another driver gene in prolactinoma, drug targeting Bcl 2 may inhibit prolactinoma. CALM1 serves a vital role in regulating a series of cellular functions through interaction with multiple target proteins (44). Calmodulin and interconnected calmodulin-regulated systems are involved in tumor growth, tumor-associated angiogenesis and metastasis (45), which is consistent with the GSEA results of the current study, which indicated that the expression profiles of prolactinomas were enriched in 'calcium signaling pathway'.
These findings suggest that CALM1 may be a potential therapeutic target for prolactinoma. In addition, the present study revealed that the genes JUN, FOS, Bcl2, STAT3, RPS27A, ACACB, INSR, ERBB2, PPP2CA, CDK2 and PTPRC are also associated with prolactinoma; these genes are potential diagnosis biomarkers, treatment targets and prognosis markers for patients with prolactinoma.

The functional analysis and enrichment of modules genes were also investigated in the present study, those modules were the most important gene clusters associated with prolactinoma, and may have vital roles in the occurrence and development mechanism of prolactinoma. The genes in module 1 were mainly associated with 'endoplasmic reticulum lumen', 'extracellular matrix organization' and 'collagen trimer', which were mainly associated with cellular structure. The endoplasmic reticulum (ER) is a major site for protein synthesis, lipid production and calcium storage in eukaryotic cells. When the endoplasmic reticulum is filled with unfolded proteins, misfolded proteins or changes in calcium concentration, ER stress (ERS) can be induced, leading to 
A



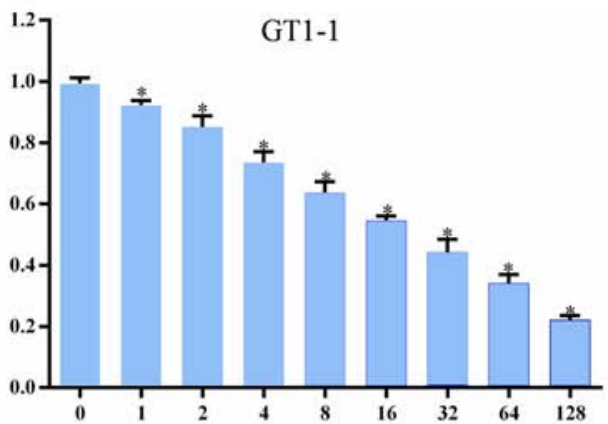

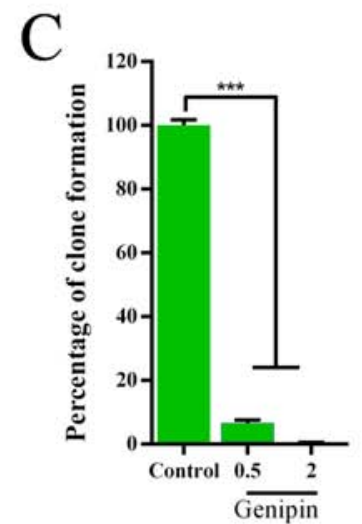

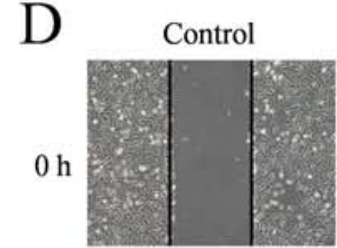

B
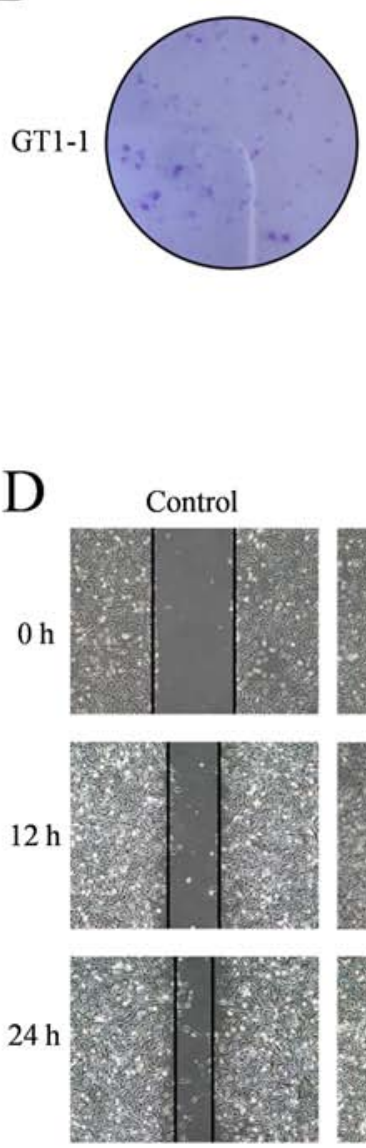

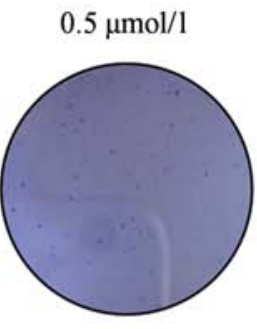

Genipin
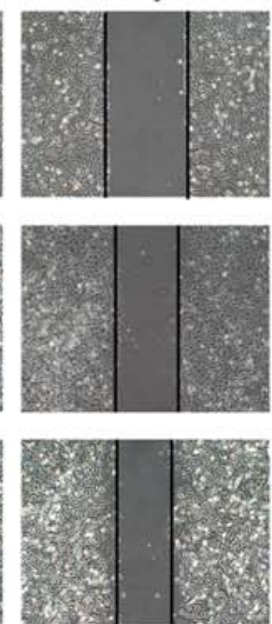

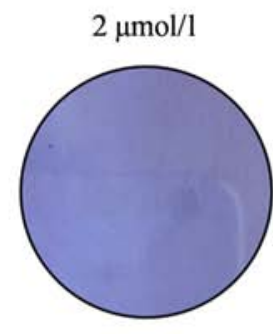

E

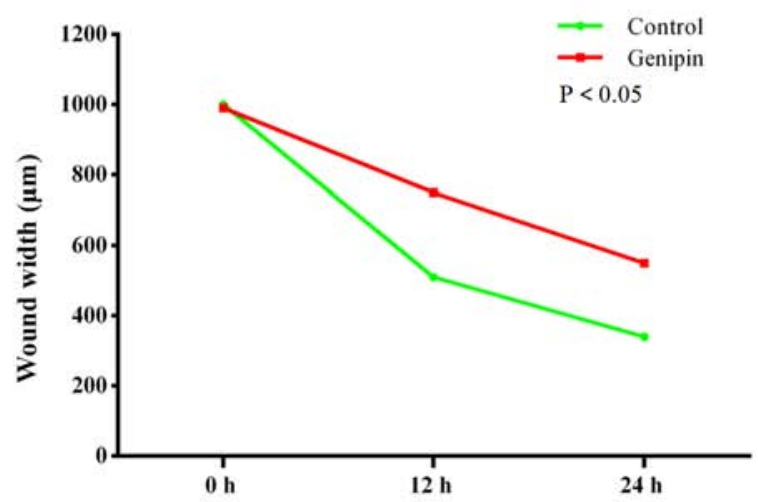

Figure 4. Anti-prolactinoma effect of Genipin (A) Cellular viability of prolactinoma cells treated with genipin. (B) Clonogenicities in Petri dishes with different dose of genipin in GT1-1 cell line. (C) Percentage of clone formation in GT1-1 cell line. (D) Scratch assay in control and genipin groups in GT1-1 cell line. (E) Wound width in control and genipin groups in GT1-1 cell line. ${ }^{*} \mathrm{P}<0.05$ and ${ }^{* * *} \mathrm{P}<0.01$ vs. Control.

apoptosis. A number of previous studies have demonstrated that ERS is associated with progression and proliferation of hepatic carcinoma and ovarian cancer $(46,47)$. The genes of module 2 and 3 were associated with 'GABAergic synapse', 'morphine addiction', 'type I interferon signaling pathway' and 'PI3K-Akt signaling pathway'. 'GABAergic synapses' regulate the activity of excitatory neurons in different brain regions. The release of neurotransmitter $\gamma$-aminobutyric acid is caused by the activation of inhibitory neurons (48). Therefore, there is a phenomenon in which excitatory neurons and inhibitory neurons co-activate during normal brain activity. The disruption of the balance between excitability and inhibition of neurons occurs in neurodegenerative and psychiatric disorders, including epilepsy, schizophrenia and Parkinson's disease (49,50). Moreover, the enriched KEGG pathways included 'morphine addiction', so it is hypothesized that taking drugs may increase the risk of prolactinoma.

The anti-prolactinoma effects of genipin were evaluated in present study with CCK8 assay, colony-forming assay and scratch assay in vitro. In CCK8 assay, the cellular viability (ratio to control) in cell lines GH3 and GT1-1 decreased with increasing drug doses of genipin. In colony-forming assay, the number and size of clonogenicities in genipin group were clearly fewer than the control group and the percentage of clone formation was significantly different in groups with different genipin doses. Those results verified that the proliferation of 

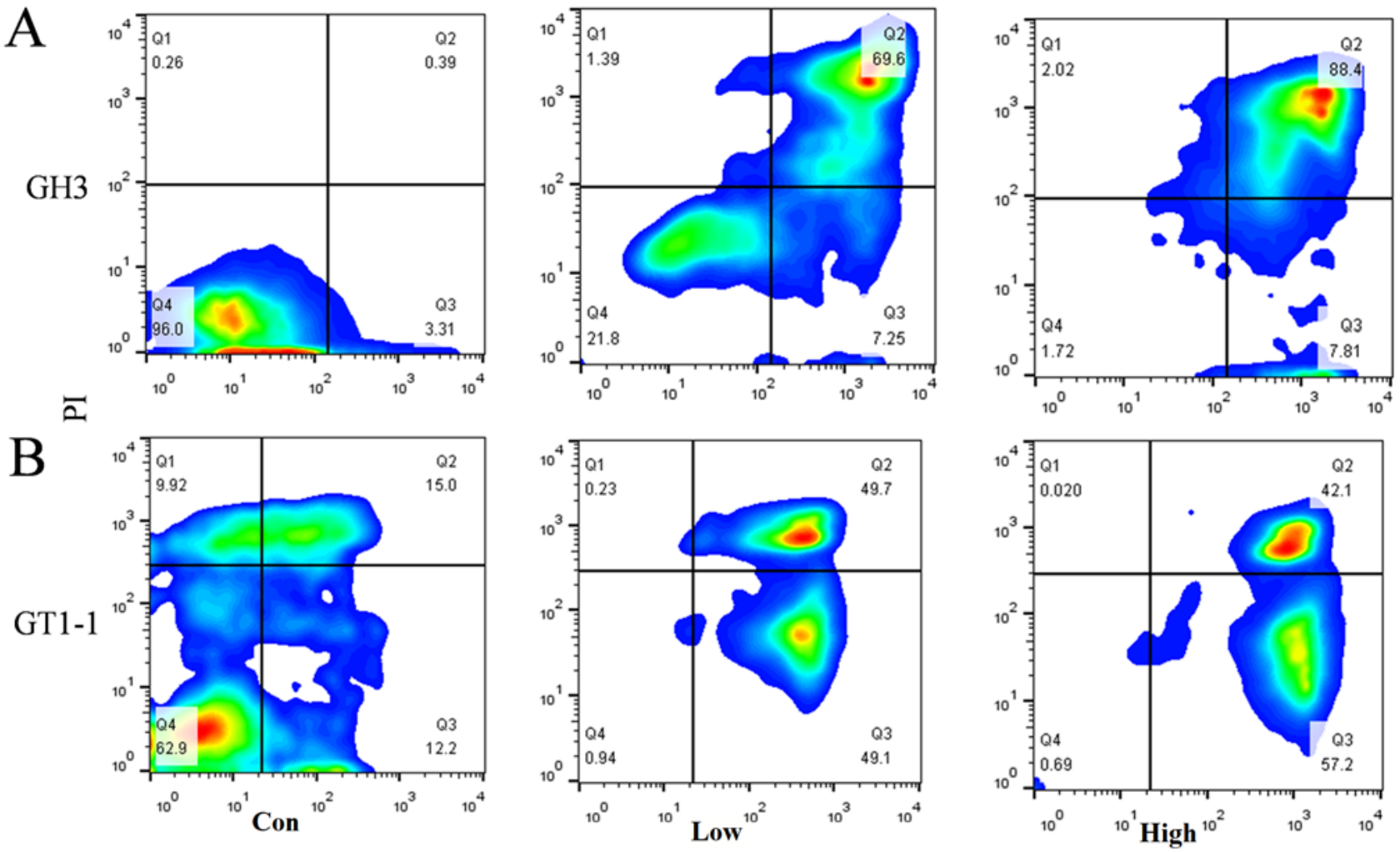

Annexin V-FITC

$\mathrm{C}$

$\mathrm{GH} 3$



$\mathrm{D}$

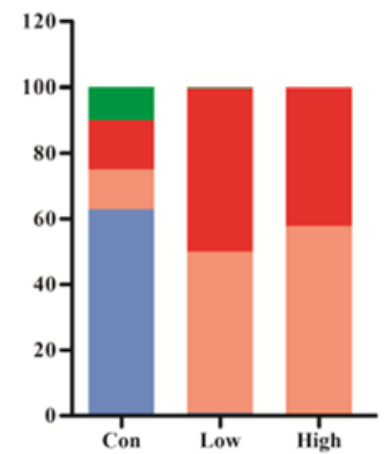

Normal cell Early apoptosis Late apoptosis Necrosis

Figure 5. (A) The distribution of cells in apoptosis with different doses of genipin in GH3 cell lines. (B) The distribution of cells in apoptosis with different doses of genipin in GT1-1 cell lines. The percentage of apoptosis cell in (C) GH3 cell lines and (D) GT1-1 cell lines treated with different doses of genipin. Normal cells were dominant in the control group; in the low and dose groups, the percentage of late and early apoptosis cells was increased (upper right quadrant is early apoptosis, upper left quadrant is late apoptosis, lower left quadrant is normal and lower right quadrant is necrosis). Con, control, Low $0.5 \mu$ mol/1 and High $2.0 \mu \mathrm{mol} / 1$ doses of genipin.

prolactinoma cells was reduced by genipin and the effects were dose-dependent. In scratch assays, the wound widths in the control group decreased over time and were smaller than the genipin group after $24 \mathrm{~h}$; this suggested that genipin reduced the migration of prolactinoma cells. Flow cytometry was performed to further investigate the anti-prolactinoma effect of genipin in prolactinoma cells, which were treated with different dose of genipin for $48 \mathrm{~h}$. The results demonstrated that the percentage of the apoptosis cells increased with the genipin dose, implying that the anti-prolactinoma effects of genipin were caused by inducing apoptosis and that this effect is dose-dependent. The absence of animal experiments was a limitation of this study, due to the lack of an ideal prolactinoma animal model; the effects of genipin in animals requires further investigation, as do the details of the mechanisms of prolactinoma.
In summary, an aggregate of $12,695,3,847$ and 5,310 DEGs were identified from datasets GSE2175, GSE26966 and GSE36314 respectively. GO and KEGG analysis showed that the enriched functions and pathways in upregulated genes were mainly related to 'spindle pole', 'oocyte meiosis' and 'nucleus'; while the downregulated genes were associated with 'signal transduction', 'cytoplasm' and 'receptor complex'. MAPK1, MYC, Bcl2, CALM1 and EGR1 were identified as main hub genes. Genes JUN, FOS, Bcl2, STAT3, RPS27A, ACACB, INSR, ERBB2, PPP2CA, CDK2 and PTPRC were revealed to be associated with prolactinoma for the first time, to the best of our knowledge. The proliferation and invasion of prolactinoma cells could be reduced by genipin via inducing apoptosis of prolactinoma cells. The anti-prolactinoma effect of genipin is dose-dependent. Genipin is a promising and potential drug for patients with prolactinoma. 


\section{Acknowledgements}

Not applicable.

\section{Funding}

The present study was supported by grants from the National Natural Science Foundation of China (grants nos. 81672505 and 81772684), the S\&T Development Planning Program of Jilin Province (grants nos. 20160101086JC, 20160 312017ZG and 20180101152JC), the Jilin Provincial Education Department '13th Five-Year' Science and Technology Project (grants no. JJKH20180191KJ) and the Interdisciplinary Innovation Project of The First Hospital of Jilin University (grants no. JDYYJC001).

\section{Availability of data and materials}

The datasets used and/or analyzed during the present study are available from the corresponding author upon reasonable request.

\section{Authors' contributions}

SZ, BW, XW and DS were major contributors in writing the manuscript, downloading datasets and conducting bio-information analysis. DL, SJ and XL performed the analysis of the results. JG, YZ and XZ performed cell experiments and contributed to figures and tables. RJ and YC supervised the study, and contributed to the experiments and data analysis.

\section{Ethics approval and consent to participate}

Not applicable.

\section{Patient consent for publication}

Not applicable.

\section{Competing interests}

The authors declare that they have no competing interests.

\section{References}

1. Theodros D, Patel M, Ruzevick J, Lim M and Bettegowda C: Pituitary adenomas: Historical perspective, surgical management and future directions. CNS Oncol 4: 411-429, 2015.

2. Louis DN, Perry A, Reifenberger G, von Deimling A, Figarella-Branger D, Cavenee WK, Ohgaki H, Wiestler OD, Kleihues P and Ellison DW: The 2016 world health organization classification of tumors of the central nervous system: A summary. Acta Neuropathol 131: 803-820, 2016.

3. Hirohata T, Ishii Y and Matsuno A: Treatment of pituitary carcinomas and atypical pituitary adenomas: A review. Neurol Med Chir (Tokyo) 54: 966-973, 2014.

4. Sinha S, Sharma BS and Mahapatra AK: Microsurgical management of prolactinomas-clinical and hormonal outcome in a series of 172 cases. Neurol India 59: 532-536, 2011.

5. Manuylova E, Calvi LM, Hastings C, Vates GE, Johnson MD, Cave WT Jr and Shafiq I: Late presentation of acromegaly in medically controlled prolactinoma patients. Endocrinol Diabetes Metab Case Rep 2016: pii: 16-0069, 2016.

6. Ghadirian H, Shirani M, Ghazi-Mirsaeed S, Mohebi S and Alimohamadi M: Pituitary apoplexy during treatment of prolactinoma with cabergoline. Asian J Neurosurg 13: 93-95, 2018.
7. Chng E and Dalan R: Pituitary apoplexy associated with cabergoline therapy. J Clin Neurosci 20: 1637-1643, 2013.

8. Nakhleh A, Shehadeh N, Hochberg I, Zloczower M, Zolotov S, Taher R and Daoud Naccache D: Management of cystic prolactinomas: A review. Pituitary 21: 425-430, 2018.

9. Zhou W, Ma C and Yan Z: Microarray data analysis reveals differentially expressed genes in prolactinoma. Neoplasma 62: 53-60, 2015.

10. Zhao L, Lin M and Wang S: Identification of human prolactinoma related genes by DNA microarray. J Cancer Res Ther 10: 544-548, 2014.

11. Zhan $X$, Wang $X$ and Cheng T: Human pituitary adenoma proteomics: New progresses and perspectives. Front Endocrinol (Lausanne) 7: 54, 2016.

12. Zhang W, Zang Z, Song Y, Yang H and Yin Q: Co-expression network analysis of differentially expressed genes associated with metastasis in prolactin pituitary tumors. Mol Med Rep 10: 113-118, 2014.

13. Faraoni EY, Camilletti MA, Abeledo-Machado A, Ratner LD, De Fino F, Huhtaniemi I, Rulli SB and Díaz-Torga G: Sex differences in the development of prolactinoma in mice overexpressing hCG $\beta$ : Role of TGF $\beta 1$. J Endocrinol 232: 535-546, 2017.

14. Ko H, Kim JM, Kim SJ, Shim SH, Ha CH and Chang HI: Induction of apoptosis by genipin inhibits cell proliferation in AGS human gastric cancer cells via Egr1/p21 signaling pathway. Bioorg Med Chem Lett 25: 4191-4196, 2015.

15. Xu Z, Zhou Y, Cao Y, Dinh TL, Wan J and Zhao M: Identification of candidate biomarkers and analysis of prognostic values in ovarian cancer by integrated bioinformatics analysis. Med Oncol 33: 130, 2016.

16. Morris DG, Musat M, Czirják S, Hanzély Z, Lillington DM, Korbonits $\mathrm{M}$ and Grossman AB: Differential gene expression in pituitary adenomas by oligonucleotide array analysis. Eur J Endocrinol 153: 143-151, 2005.

17. Michaelis KA, Knox AJ, Xu M, Kiseljak-Vassiliades K, Edwards MG, Geraci M, Kleinschmidt-DeMasters BK, Lillehei KO and Wierman ME: Identification of growth arrest and DNA-damage-inducible gene beta (GADD45beta) as a novel tumor suppressor in pituitary gonadotrope tumors. Endocrinology 152: 3603-3613, 2011.

18. Tong Y, Zheng Y, Zhou J, Oyesiku NM, Koeffler HP and Melmed S: Genomic characterization of human and rat prolactinomas. Endocrinology 153: 3679-3691, 2012.

19. Abdul Aziz NA, Mokhtar NM, Harun R, Mollah MM, Mohamed Rose I, Sagap I, Mohd Tamil A, Wan Ngah WZ and Jamal R: A 19-Gene expression signature as a predictor of survival in colorectal cancer. BMC Med Genomics 9: 58, 2016.

20. Huang DW, Sherman BT and Lempicki RA. Systematic and integrative analysis of large gene lists using DAVID Bioinformatics Resources. Nat Protoc 4: 44-57, 2009.

21. Shannon P, Markiel A, Ozier O, Baliga NS, Wang JT, Ramage D, Amin N, Schwikowski B and Ideker T: Cytoscape: A software environment for integrated models of biomolecular interaction networks. Genome Res 13: 2498-2504, 2003.

22. Franken NA, Rodermond HM, Stao J, Haveman J and van Bree C: Clonogenic assay of cells in vitro. Nat Protoc 1: 2315-2319, 2006.

23. Cooper O, Mamelak A, Bannykh S, Carmichael J, Bonert V, Lim S, Cook-Wiens G and Ben-Shlomo A: Prolactinoma ErbB receptor expression and targeted therapy for aggressive tumors. Endocrine 46: 318-327, 2014.

24. Castro-Ferreira R, Neves JS, Ladeiras-Lopes R, Leite-Moreira AM, Neiva-Sousa M, Almeida-Coelho J, Ferreira-Martins J and F Leite-Moreira A: Revisiting the slow force response: The role of the PKG signaling pathway in the normal and the ischemic heart. Rev Port Cardiol 33: 493-499, 2014.

25. Hou J and Kang YJ: Regression of pathological cardiac hypertrophy: Signaling pathways and therapeutic targets. Pharmacol Ther 135: 337-354, 2012.

26. Babykutty S, Suboj P, Srinivas P, Nair AS, Chandramohan K and Gopala S: Insidious role of nitric oxide in migration/invasion of colon cancer cells by upregulating MMP-2/9 via activation of cGMP-PKG-ERK signaling pathways. Clin Exp Metastasis 29: 471-492, 2012.

27. Browning DD, Kwon IK and Wang R: cGMP-dependent protein kinases as potential targets for colon cancer prevention and treatment. Future Med Chem 2: 65-80, 2010.

28. Riquelme I, Tapia O, Espinoza JA, Leal P, Buchegger K, Sandoval A, Bizama C, Araya JC, Peek RM and Roa JC: The Gene Expression Status of the PI3K/AKT/mTOR pathway in gastric cancer tissues and cell lines. Pathol Oncol Res 22: 797-805, 2016. 
29. Guo H, German P, Bai S, Barnes S, Guo W, Qi X, Lou H, Liang J, Jonasch E, Mills GB and Ding Z: The PI3K/AKT pathway and renal cell carcinoma. J Genet Genomics 42: 343-353, 2015.

30. Wu Y, Yuan M, Su W, Zhu M, Yao X, Wang Y, Qian H, Jiang L, Tao Y, Wu M, et al: The constitutively active PKG II mutant effectively inhibits gastric cancer development via a blockade of EGF/EGFR-associated signalling cascades. Ther Adv Med Oncol 10: 1758834017751635, 2018.

31. Chauvin TR, Herndon MK and Nilson JH: Cold-shock-domain protein A (CSDA) contributes posttranscriptionally to gonadotropin-releasing hormone-regulated expression of Egr1 and indirectly to Lhb. Biol Reprod 86: 53, 2012.

32. Kim ES, Jeong CS and Moon A: Genipin, a constituent of Gardenia jasminoides Ellis, induces apoptosis and inhibits invasion in MDA-MB-231 breast cancer cells. Oncol Rep 27: 567-572, 2012.

33. Wang R, MoYung KC, Zhao YJ and Poon K: A mechanism for the temporal potentiation of genipin to the cytotoxicity of cisplatin in colon cancer cells. Int J Med Sci 13: 507-516, 2016.

34. Santarpia L, Lippman SM and El-Naggar AK: Targeting the MAPK-RAS-RAF signaling pathway in cancer therapy. Expert Opin Ther Targets 16: 103-119, 2012.

35. Hu L, Wu H, Wan X, Liu L, He Y, Zhu L, Liu S, Yao H and Zhu Z: MicroRNA-585 suppresses tumor proliferation and migration in gastric cancer by directly targeting MAPK1. Biochem Biophys Res Commun 499: 52-58, 2018.

36. Li C, Sun Z, Gui S, Liu F and Zhang Y: Effects of fulvestrant, an estrogen receptor antagonist, on MMQ cells and its mechanism. Neuro Endocrinol Lett 30: 268-274, 2009.

37. Tu WB, Helander S, Pilstål R, Hickman KA, Lourenco C, Jurisica I, Raught B, Wallner B, Sunnerhagen M and Penn LZ: Myc and its interactors take shape. Biochim Biophys Acta 1849: 469-483, 2015.

38. Kim EY, Kim A, Kim SK and Chang YS: MYC expression correlates with PD-L1 expression in non-small cell lung cancer. Lung Cancer 110: 63-67, 2017.

39. Li S, Lin P, Young KH, Kanagal-Shamanna R, Yin CC and Medeiros LJ: MYC/Bcl2 double-hit high-grade B-cell lymphoma. Adv Anat Pathol 20: 315-326, 2013.

40. Zhang J, Yao YH, Li BG, Yang Q, Zhang PY and Wang HT: Prognostic value of pretreatment serum lactate dehydrogenase level in patients with solid tumors: A systematic review and meta-analysis. Sci Rep 5: 9800, 2015.
41. Jagani H, Kasinathan N, Meka SR and Josyula VR: Antiapoptotic Bcl-2 protein as a potential target for cancer therapy: A mini review. Artif Cells Nanomed Biotechnol 44: 1212-1221, 2016.

42. Okubo S, Kurebayashi J, Otsuki T, Yamamoto Y, Tanaka K and Sonoo H: Additive antitumour effect of the epidermal growth factor receptor tyrosine kinase inhibitor gefitinib (Iressa, ZD1839) and the antioestrogen fulvestrant (Faslodex, ICI 182,780) in breast cancer cells. Br J Cancer 90: 236-244, 2014.

43. Geng X, Ma L, Li Z, Li Z, Li J, Li M, Wang Q, Chen Z and Sun Q Bromocriptine induces autophagy-dependent cell death in pituitary adenomas. World Neurosurg 100: 407-416, 2017.

44. Toutenhoofd SL, Foletti D, Wicki R, Rhyner JA, Garcia F, Tolon R and Strehler EE: Characterization of the human CALM2 calmodulin gene and comparison of the transcriptional activity of CALM1, CALM2 and CALM3. Cell Calcium 23: 232-238, 1998.

45. Berchtold MW and Villalobo A: The many faces of calmodulin in cell proliferation, programmed cell death, autophagy, and cancer. Biochim Biophys Acta 1843: 398-435, 2014.

46. Hughes A, Oxford AE, Tawara K, Jorcyk CL and Oxford JT: Endoplasmic reticulum stress and unfolded protein response in cartilage pathophysiology; contributing factors to apoptosis and osteoarthritis. Int J Mol Sci 18: pii: E665, 2017.

47. Tang MK and Wong AS: Exosomes: Emerging biomarkers and targets for ovarian cancer. Cancer Lett 367: 26-33, 2015.

48. Siegel JM: The neurotransmitters of sleep. J Clin Psychiatry 65 (Suppl 16): S4-S7, 2004.

49. Tyson JA and Anderson SA: GABAergic interneuron transplants to study development and treat disease. Trends Neurosci 37: 169-177, 2014.

50. Southwell DG, Nicholas CR, Basbaum AI, Stryker MP, Kriegstein AR, Rubenstein JL and Alvarez-Buylla A: Interneurons from embryonic development to cell-based therapy. Science 344: 1240622, 2014

This work is licensed under a Creative Commons Attribution-NonCommercial-NoDerivatives 4.0 International (CC BY-NC-ND 4.0) License. 